\title{
Fatores que dificultam a aplicação do processo de enfermagem pelos enfermeiros da atenção primária à saúde
}

\author{
Factors that hider the application of the nursing process by primary health care nurses \\ Factores que dificultan la aplicación del processo de enfermaría por los enfermeros de la \\ atención primaria a la salud
}

Evelin Reis Macedo ${ }^{1 *}$, Ana Carolina Mendonça Basílio ${ }^{1}$, Bárbara Jordânia Rodrigues Silva ${ }^{1}$, Brenda Domingos Vitorino Santos ${ }^{1}$, Clara Rodrigues de Andrade ${ }^{1}$, Gilberto de Souza ${ }^{1}$, Rafaela Dias Pardini ${ }^{1}$.

\section{RESUMO}

Objetivo: Identificar os motivos pelos quais os profissionais de enfermagem da Atenção Primária à Saúde (APS) não aplicam o Processo de Enfermagem (PE) adequadamente. Métodos: Trata-se de uma revisão integrativa da literatura que visou analisar os fatores que interferem na execução do PE. Para o estudo, utilizou-se artigos publicados nos anos de 2016 a 2020, coletados na BVS, a qual abrange bases de dados como LILACS, MEDLINE, SCIELO e BDENF, que estivessem em língua portuguesa e disponíveis eletronicamente na íntegra. Após as buscas nas bases selecionadas e atendendo os descritores e critérios pré-definidos pelos autores, foram selecionados 15 artigos para a amostra final. Resultados: As dificuldades que se apresentaram com maiores frequências foram as seguintes: falta de capacitação para aplicação do PE $(30,7 \%)$, sobrecarga de trabalho $(26,8 \%)$, falta de espaços físicos para realizar a consulta de enfermagem (CE) $(11,4 \%)$ e desvalorização do profissional enfermeiro $(7,7 \%)$. Os resultados do presente artigo evidenciaram diversos aspectos que dificultam a aplicação do PE pelos enfermeiros na APS. Considerações finais: Diante dessas informações, é possível traçar planos e estratégias para solucionar tais adversidades, a fim de estimular o uso dessa ferramenta tão importante para o desenvolvimento científico da profissão.

Palavras-chave: Enfermagem, Processo de enfermagem, Atenção primária à saúde.

\begin{abstract}
Objective: Identify the reasons why nursing professionals in Primary Health Care (PHC) don't apply the Nursing Process (NP) properly. Methods: It is an integrative literature review that aimed to analyze the factors that interfere in the execution of NP. For the study, were used articles published from 2016 to 2020, collected in BVS, which cover data base such as LILACS, MEDLINE, SCIELO and BDENF, that were in Portuguese language and available electronically in full. After searches in selected databases and attending the descriptors and criteria predefined by the authors, were selected 15 articles for the final sample. Results: The most frequent difficulties were the following: lack of training to apply the NP $(30,7 \%)$, overwork $(26,8 \%)$, lack of physical spaces to carry out the nursing consultation $(11,4 \%)$ and depreciation of the professional nurse $(7,7 \%)$. The results of this article evidenced several aspects that hinder the application of NP by nurses in PHC. Final considerations: Given this information, it is possible to devise plans and strategies to solve such adversities, in order to encourage the use of this very important tool for the scientific development of the profession.
\end{abstract}

Keywords: Nursing, Nursing process, Primary health care.

\section{RESUMEN}

Objetivos: Identificar los motivos por los que los profesionales de enfermería de la Atención Primaria a la Salud (APS) no aplican el Proceso de Enfermería (PE) correctamente. Métodos: Se trata de una revisión integradora de la literatura que buscó analizar los factores que interferen en la ejecución del PE. Para el

${ }^{1}$ Universidade Do Estado de Minas Gerais (UEMG), Divinópolis - MG. *E-mail: ev.reis23@yahoo.com.br 
estudio, se utilizó articulos publicados en los años 2016 a 2020, recogidos en la BVS, que reune base de datos como LILACS, MEDLINE, SCIELO y BDENF, desde que estuvieran en portugues y disponibles integralmente en medio electrónico. Tras las búsquedas en las fuentes seleccionadas, satisfaciendo los descriptores y criterios predefinidos por los autores, fueron seleccionados 15 articulos para la muestra final. Resultados: Las dificultades que se presentaron más frecuentemente fueron las siguentes: ausencia de capacitación para la aplicación del PE $(30,7 \%)$, sobrecarga de trabajo $(26,8 \%)$, ausencia del espacio físico necesario para el suceso de la consulta de enfermería (CE) $(11,4 \%)$ y desvaluación del profesional de enfermería $(7,7 \%)$. Los resultados de este articulo demuestran varios aspectos que dificultan la aplicación del PE por los enfermeros en la APS. Consideraciones finales: Ante estas informaciones, es posible desarrollar planes y estrategias para solucionar tales adversidades con vistas a estimular el uso de esa herramienta tan relevante para el desarrollo cientifico de la profesión.

Palabras clave: Enfermería, Proceso de enfermería, Atención primaria a la salud.

\section{INTRODUÇÃO}

A Atenção Primária à Saúde (APS) desempenha função primordial no cenário brasileiro, considerada como o primeiro nível de assistência, a mesma é vista como a porta de entrada para o Sistema Único de Saúde (SUS) (PIOVESAN G, et al., 2017). A APS caracteriza-se por um conjunto de atividades de saúde individuais, familiares e coletivas que visam a prevenção, promoção, proteção e vigilância em saúde, realizada através de equipe multiprofissional (BRASIL, 2017).

Nesse contexto, o papel do enfermeiro na APS está diretamente relacionado com uma atuação voltada para o cenário da Saúde Coletiva, com o intuito de cuidar dos seres humanos, conhecendo e interpretando a realidade de saúde de uma população, para nela intervir e, posteriormente, reinterpretá-la (CORREA VAF, et al., 2018).

O enfermeiro, sendo integrante da equipe de saúde da APS, realiza ações de prevenção e promoção da saúde, diagnóstico, tratamento, reabilitação e redução de danos. Tem como propósito ofertar assistência à saúde de qualidade para os indivíduos e coletividades (GOMES PAR, et al., 2019).

Dentre as funções desempenhadas pelo enfermeiro na APS, sabe-se que o Processo de Enfermagem (PE) é uma atividade privativa desta categoria. O PE pode ser compreendido como um importante instrumento teórico-metodológico que direciona os profissionais em suas práticas diárias, com o intuito de detectar situações no processo saúde-doença, bem como oferecer condições para intervenções na realidade do indivíduo, família e comunidade (SANTOS KC, et al., 2020).

Ademais, o PE pode ser visto como a interação de atividades sistematizadas que proporciona a organização da assistência de enfermagem. Configura-se como uma abordagem ética e humanizada que busca a resolução de dilemas, visando suprir as demandas de saúde apresentadas pelos usuários, além de guiar o caminho de possíveis intervenções que possam ser empregadas (MARCHIORI GRS, et al., 2018).

Visto a importância desta ferramenta, e a necessidade de implementação do PE como guia norteador da assistência de enfermagem, o Conselho Federal de Enfermagem (COFEN) divulgou a Resolução 358/2009, a qual aborda sobre o Processo de Enfermagem, incluindo a forma de aplicação e suas etapas. Salienta-se que o mesmo deve ser realizado de modo deliberado e sistemático, em todos os níveis de atenção em que ocorrem o cuidado de Enfermagem, tanto de caráter público como privado (COFEN, 2009).

O PE é composto por cinco etapas inter-relacionadas, interdependentes e recorrentes, sendo elas: a coleta de dados, diagnóstico de enfermagem (DE), planejamento, implementação e a avaliação de enfermagem. A primeira fase (coleta de dados) é considerada eixo primordial para o desenvolvimento da Sistematização da Assistência de Enfermagem (SAE), visto que a obtenção de informações fidedignas favorecerá a elaboração de um plano de cuidado que seja mais condizente com a realidade do usuário. Caso ocorra o oposto, e os dados colhidos sejam infundados, a construção do processo se tornará falha e, consequentemente, a assistência prestada será ineficaz (SANTOS KC, et al., 2020). 
$\mathrm{Na}$ assistência ao usuário com doença crônica, por exemplo, o enfermeiro tem função relevante na promoção e manutenção da saúde e a prevenção de complicações através da Consulta de Enfermagem (CE). Assim, faz-se necessário potencializar o trabalho das equipes da APS por meio do seu conhecimento científico e na gestão dos serviços de saúde. Contudo, estudos revelam que na rotina dos trabalhos na APS, a realização da CE ainda é fragilizada e fragmentada devido à inúmeros fatores (MOTA BAM, et al., 2019).

A implantação da SAE e do PE é uma deliberação legal, porém não é praticada de maneira satisfatória. Na APS, a efetivação da SAE demonstra ser bem iniciante. Um estudo aponta a escassez de artigos sobre SAE ou PE na APS, pois a maioria das pesquisas encontradas ocorreu no âmbito hospitalar (RIBEIRO GC e PADOVEZE MC, 2018). No entanto, o uso do PE incentiva a continuidade do cuidado, bem como é uma ferramenta de assistência de enfermagem que propicia o reconhecimento profissional, otimiza a prática nos serviços e favorece o desenvolvimento da enfermagem como ciência (BEZERRIL MS, et al., 2021).

Diante disso, este estudo se justifica pela necessidade de investigar quais são os fatores que dificultam a aplicação do PE na APS pelos enfermeiros. Esta pesquisa tem como objetivo identificar os motivos pelos quais os profissionais de enfermagem da APS não aplicam o PE adequadamente.

\section{MÉTODOS}

Trata-se de uma revisão integrativa da literatura que seguiu as seguintes fases: identificação do tema e seleção da hipótese; estabelecimento de critérios para inclusão e exclusão de estudos; definição das informações a serem extraídas dos estudos escolhidos; avaliação dos estudos incluídos; interpretação dos resultados e apresentação dos mesmos. A revisão integrativa da literatura é um método que visa sintetizar resultados obtidos através da pesquisa de um tema específico, de maneira organizada e ampla (SOUSA LMM, et al., 2018).

A questão norteadora da pesquisa foi seguinte: Quais fatores dificultam a aplicação do PE por enfermeiros que atuam na APS?

A busca na literatura foi realizada entre dezembro de 2020 e março de 2021. Para o estudo optou-se pela busca na Biblioteca Virtual de Saúde (BVS) que abrange as seguintes bases de dados: Lilacs (Literatura Latino- Americana e do Caribe em Ciências da Saúde), Medline (Medical Literature Analysis and Retrieval Sistem on-line) BDENF (Base de Dados de Enfermagem) e Scielo (Scientific Eletronic Library Online). Foram utilizados os seguintes descritores: atenção primária à saúde, enfermagem e processo de enfermagem, todos validados no DeCS (Descritores em Ciências da Saúde).

Os critérios para inclusão dos estudos foram artigos sobre a temática aplicação do processo de enfermagem na APS publicados em periódicos nacionais, a partir do ano de 2016 a 2020, que englobam os objetivos propostos; em língua portuguesa; e disponíveis eletronicamente na íntegra. Os critérios de exclusão foram: livros, teses, dissertações e estudos que não abordassem a temática proposta.

Inicialmente a seleção dos artigos foi baseada no processo da leitura dos títulos e resumos para exclusão de publicações duplicadas e que não atendiam ao tema. Posteriormente os artigos selecionados foram lidos na íntegra.

\section{RESULTADOS}

Após a pesquisa nas bases de dados e atendendo aos critérios pré-estabelecidos pelas autoras, realizouse a leitura dos títulos e resumos dos estudos encontrados, chegando ao número de 30 artigos, sendo que 1 foi excluído por estar duplicado. Em seguida, efetuou-se a leitura crítica de todos os artigos na íntegra, com o intuito de que a pergunta norteadora fosse respondida. A partir dessa etapa foram selecionados 15 artigos.

Para melhor análise e interpretação dos resultados, elaborou-se o Quadro 1. Todos os artigos selecionados foram publicados entre os anos de 2016 a 2021. A revista Enfermagem em Foco foi o periódico de destaque de publicações sobre o tema em questão, totalizando 3 artigos. 
Quadro 1 - Informações dos artigos selecionados a respeito dos fatores que dificultam a aplicação do PE pelos enfermeiros na APS, 2016 -2021.

\begin{tabular}{|c|c|c|}
\hline $\mathbf{N}$ & Autores (Ano) & $\begin{array}{ll} & \text { Principais achados }\end{array}$ \\
\hline 1 & $\begin{array}{c}\text { AMARAL IBST e SILVA ALA } \\
(2021)\end{array}$ & $\begin{array}{l}\text { Trata-se de uma pesquisa descritiva de abordagem qualitativa cujos dados foram colhidos através de trabalho de campo em quatro } \\
\text { unidades básicas de saúde na cidade do Rio de Janeiro. Os resultados obtidos apontam para uma tendência à realização de } \\
\text { abordagens verticais ao indivíduo, com foco nos programas do ministério da saúde, e no modelo biomédico- flexineriano de atenção, } \\
\text { além de revelar repetição contínua de interrupções ao longo das consultas, fragmentando os encontros Enfermeiro-paciente. Sendo } \\
\text { assim, defende-se uma reorganização do processo de trabalho de modo que o enfermeiro permita o protagonismo do usuário. }\end{array}$ \\
\hline 2 & ROSA APL, et al. (2020) & $\begin{array}{l}\text { Trata-se de uma pesquisa ação, baseada no referencial metodológico de Thiollent. Participaram } 10 \text { enfermeiras que realizavam } \\
\text { consulta de enfermagem na APS em Chapeco-SC. Os principais desafios para efetivação do PE estão relacionados ao processo de } \\
\text { trabalho do enfermeiro, sobrecarga, acumulo de funções administrativas e assistenciais, falta de tempo, déficit de recursos humanos } \\
\text { e materiais, grande demanda de usuários nos serviços de saúde. O estudo demonstrou a necessidade de adequações no processo } \\
\text { de trabalho do enfermeiro, de forma a permitir uma assistência de qualidade a mulher, aprimorando a prática clínica e os registros } \\
\text { de enfermagem. }\end{array}$ \\
\hline 3 & SIEGA CK, et al. (2020) & $\begin{array}{l}\text { Pesquisa-ação com } 15 \text { enfermeiros atuantes na Atenção Primária à Saúde. O estudo revelou três categorias: história pregressa e } \\
\text { atual da criança e sua família; Etapas para a implementação do cuidado sistematizado e Dificuldades para realizar a Consulta do } \\
\text { Enfermeiro em puericultura na Atenção Primária à Saúde. Desta forma, em suas vivências os enfermeiros relatam dificuldades que } \\
\text { culminam na fragmentação da Consulta, entretanto, este instrumento é uma oportunidade para conhecer a criança e família na sua } \\
\text { integralidade. }\end{array}$ \\
\hline 4 & ZANATTA EA, et al. (2020) & $\begin{array}{l}\text { Estudo exploratório, descritivo com abordagem qualitativa, realizado com dez enfermeiros por meio de entrevista realizada em julho } \\
\text { de 2018. A análise dos dados deu origem a duas categorias: Dificuldades na intercomunicação entre enfermeiros e famílias de } \\
\text { crianças haitianas e Dificuldades culturais relacionadas ao cuidado da criança haitiana. Os resultados revelam dificuldades na } \\
\text { realização da Consulta de Enfermagem às crianças, especialmente relacionadas à intercomunicação, pela língua falada pelos } \\
\text { haitianos, e pelas questões culturais que permeiam e embasam o cuidado à criança pelas famílias. }\end{array}$ \\
\hline 5 & MOTA BAM, et al. (2019) & $\begin{array}{l}\text { Ensaio clínico não-controlado realizado em uma Estratégia Saúde da Família de Minas Gerais, onde } 14 \text { participantes foram } \\
\text { acompanhados por meio da assistência sistematizada de enfermagem entre os meses de outubro de } 2016 \text { e setembro de } 2017 . \\
\text { Verificou-se uma diferença estatisticamente significativa na adesão ao tratamento da hipertensão arterial sistêmica após as } \\
\text { intervenções de enfermagem, que foram realizadas individualmente e coletivamente. A assistência sistematizada de enfermagem } \\
\text { pode beneficiar pessoas diagnosticadas com hipertensão arterial sistêmica na atenção primária em saúde. Faz-se necessário o } \\
\text { fortalecimento da utilização do processo de enfermagem e da identidade do enfermeiro no cuidado das condições crônicas. }\end{array}$ \\
\hline 6 & $\begin{array}{l}\text { SOMARIVA VCA, et al. } \\
\text { (2019) }\end{array}$ & $\begin{array}{l}\text { Trata-se de um estudo transversal, com abordagem quantitativa, realizado com } 76 \text { profissionais de enfermagem, por meio de } \\
\text { questionário com } 66 \text { questões objetivas. Os participantes demonstraram ter conhecimento, capacidade de identificação dos } \\
\text { benefícios e elementos dificultadores ou facilitadores da SAE, contudo estes ainda apresentam dificuldades para aplicar a SAE em } \\
\text { seu local de trabalho. }\end{array}$ \\
\hline 7 & MATA LRF, et al. (2019) & $\begin{array}{l}\text { Estudo descritivo que utilizou como fontes de informação e-mails respondidos e pareceres técnicos publicados no período de agosto } \\
\text { de } 2015 \text { a agosto de } 2016 \text {. Considerando as áreas temáticas, } 436 \text { consultas eram referentes a área gerencial e assistencial, } 267 \text { a } \\
\text { atenção primaria e } 14 \text { a sistematização da assistência de enfermagem. Os resultados refletem que os pontos mais questionados } \\
\text { referem-se a questões técnicas do exercício profissional e estimulam a reflexão sobre possíveis posicionamentos técnicos a serem } \\
\text { defendidos pelos Conselhos de Enfermagem. }\end{array}$ \\
\hline
\end{tabular}




\begin{tabular}{|c|c|c|}
\hline $\mathbf{N}$ & Autores (Ano) & Principais achados \\
\hline 8 & $\begin{array}{c}\text { RIBEIRO GC e PADOVEZE } \\
\text { MC (2018) }\end{array}$ & $\begin{array}{l}\text { Estudo quantitativo, descritivo-exploratório, realizado em uma Unidade Básica de Saúde no interior de São Paulo. Verificou-se que } \\
\text { a implantação da Sistematização da Assistência de Enfermagem e do processo de enfermagem ainda é incipiente nas Unidades } \\
\text { Básicas de Saúde. Sendo assim, é necessário interesse institucional para a viabilização da implantação. O estudo pode contribuir } \\
\text { para a efetiva implantação nesta unidade e apontar diretrizes para a sua implementaça em outros contextos similares. }\end{array}$ \\
\hline 9 & KAHL C, et al. (2018) & $\begin{array}{l}\text { Pesquisa qualitativa ancorada na Teoria Fundamentada nos Dados. O estudo evidenciou as ações e interações ocorridas na prática } \\
\text { clínica do enfermeiro no âmbito da APS, dentre as quais se destacam a sistematização da assistência na consulta de enfermagem. } \\
\text { A consulta de enfermagem revela-se como importante meio para o desenvolvimento da prática clínica pautada no princípio da } \\
\text { integralidade e na prática baseada em evidência, permitindo um exercício sistematizado capaz de contribuir para a melhoria da } \\
\text { qualidade da assistência prestada. }\end{array}$ \\
\hline 10 & COSTA AS, et al. (2018) & $\begin{array}{l}\text { Trata-se de um estudo descritivo de abordagem qualitativa, com base na Análise de Conteúdo de Bardin. As respostas agrupadas } \\
\text { e organizadas originaram duas categorias: (1) O uso da SAE pelo enfermeiro na Atenção Básica em Saúde: potencialidades e } \\
\text { limitações; (2) Aplicação das etapas do Processo de Enfermagem: parcialidade e desconhecimento. Os resultados revelaram a } \\
\text { parcialidade na aplicação do PE no dia a dia do enfermeiro e a necessidade de empoderamento do enfermeiro quanto à sua } \\
\text { identidade e prática profissional. }\end{array}$ \\
\hline 11 & LOWEN IMV, et al. (2017) & $\begin{array}{l}\text { Estudo qualiquantitativo, tendo sido entrevistados } 32 \text { enfermeiros gerenciais e assistenciais e coletados dados documentais de } \\
\text { relatórios públicos de produção de consultas dos enfermeiros de } 2010 \text { a } 2014 \text {, num município sul-brasileiro. Na classe pratica } \\
\text { assistencial inovadora, associada a sensibilização para a mudança, relacionada a implantação da Estratégia Saúde da Família, seus } \\
\text { desafios e fortalezas, foram identificadas as subclasses: reorganização das agendas, consulta do enfermeiro, reestruturação física } \\
\text { das UBS e consulta compartilhada. O protagonismo do cuidado precisa ser discutido nos diversos espaços a fim de que cada } \\
\text { profissional desempenhe seu papel com competência e resolutividade. }\end{array}$ \\
\hline 12 & SILVEIRA V, et al. (2016) & $\begin{array}{l}\text { Estudo descritivo, exploratório, transversal, de abordagem qualitativa, realizado com } 12 \text { acadêmicos de enfermagem. Identificaram- } \\
\text { se três categorias, a saber: Implantação e uso da SAE são inadequados; Pouco interesse dos enfermeiros da ESF em aplicar a } \\
\text { SAE; Dificuldade de aplicar a SAE na saúde da família pela escassez de diagnóstico de enfermagem para a família. A Sistematização } \\
\text { da Assistência de Enfermagem foi apresentada pelos acadêmicos, no contexto do ensino clínico, de maneira falha, abstrata no } \\
\text { momento da prática, no entanto, a maior dificuldade apresentada foi em aplicar os diagnósticos de enfermagem. }\end{array}$ \\
\hline 13 & GANDOLFI M, et al. (2016) & $\begin{array}{l}\text { Estudo descritivo, tipo relato de experiência, com cinco acamados, entre } 22 \text { e } 92 \text { anos de idade, no município de Chapecó-SC. } \\
\text { Foram identificadas situaç̃̃es que requeriam manutenção, ajustamento ou repadronização de cuidados culturalmente definidos. A } \\
\text { Sistematização da Assistência de Enfermagem apoiada na teoria do cuidado transcultural proporcionou melhorias à saúde do } \\
\text { acamado e da sua família, motivando a adesão dos cuidadores ao plano de cuidados. }\end{array}$ \\
\hline 14 & ADAMY EK, et al. (2016) & $\begin{array}{l}\text { Estudo de abordagem qualitativa, exploratório e descritivo. Os enfermeiros reconhecem na sua formação conteúdos sobre } \\
\text { anamnese e exame físico e valorizam o conhecimento científico para avaliar o usuário. Têm pouco contato com essa tecnologia } \\
\text { após a graduação, em especializações ou processos de educação permanente. Percebe-se a necessidade de enfatizar o conteúdo } \\
\text { durante a formação; proporcionar condições para sua aplicabilidade e fomentar processos de educação permanente. }\end{array}$ \\
\hline 15 & $\begin{array}{l}\text { SILVA KM e SANTOS SMA } \\
(2016)\end{array}$ & $\begin{array}{l}\text { Pesquisa qualitativa, convergente-assistencial, cujos dados foram coletados entre maio e junho de } 2012 \text {. A consulta de enfermagem } \\
\text { é uma importante ferramenta de educação em saúde, favorece o vínculo profissional e usuário, possibilita o desenvolvimento da } \\
\text { autonomia e independência profissional. Apesar do reconhecimento do papel da consulta de enfermagem na práxis dos enfermeiros } \\
\text { ainda se verifica entraves para sua execução. }\end{array}$ \\
\hline
\end{tabular}

Fonte: Macedo ER, et al., 2022. 
Após a leitura na íntegra dos artigos, foram criadas categorias baseadas nas dificuldades que os enfermeiros encontram para a aplicação do PE na APS que estão detalhadas na Tabela 1. As dificuldades que apresentaram maiores frequências foram: falta de capacitação para aplicação do PE (30,7\%), sobrecarga de trabalho $(26,8 \%)$, falta de espaços físicos para realizar a consulta de enfermagem (CE) $(11,4 \%)$, desvalorização do profissional enfermeiro $(7,7 \%)$.

Tabela 1 - Fatores que dificultam a aplicação do PE pelos enfermeiros na APS, 2016-2021.

\begin{tabular}{lcc}
\hline Dificuldades para aplicar o PE & $\mathbf{n}$ & $\%$ \\
\hline Sobrecarga de trabalho & 7 & 26,8 \\
Falta de tempo & 1 & 3,9 \\
Falta de capacitação para aplicar o PE & 8 & 30,7 \\
Falta de rotina para aplicar o PE & 1 & 3,9 \\
Diferença cultural/idioma de pacientes estrangeiros & 1 & 3,9 \\
Desvalorização do profissional enfermeiro & 2 & 7,7 \\
Dificuldade em encontrar um DE adequado para o problema & 1 & 3,9 \\
de saúde identificado & 3 & 11,4 \\
Falta de espaços físicos para realizar a CE & 1 & 3,9 \\
Falta de apoio e incentivo da gestão & 1 & 3,9 \\
Resistência da equipe em aderir o PE na rotina de trabalho & & \\
\hline
\end{tabular}

Fonte: Macedo ER, et al., 2022.

\section{DISCUSSÃO}

Após a apresentação dos resultados, nota-se que os enfermeiros da APS enfrentam diversas dificuldades para aplicar o PE. Embora seja evidente a importância do uso do processo de enfermagem por parte dos enfermeiros em sua prática clínica, e os benefícios do mesmo durante a prestação da assistência ao paciente, além da sua determinação legal, observa-se a existência de muitas adversidades no cenário brasileiro que impossibilitam sua aplicação. Em algumas circunstâncias, as práticas de implementação do PE têm acontecido de forma introvertida, sendo realizadas apenas algumas etapas, em outros momentos se restringem a outros níveis de atenção (SILVA KM e SANTOS SMA, 2016).

Sobre a falta de capacitação, evidencia-se tal demanda mesmo depois de 50 anos do início da aplicação do PE no Brasil. A utilização do PE requer conhecimento técnico-científico e para sua execução é preciso que os enfermeiros conheçam as etapas que o constitui, bem como aplicá-lo na prática. O conhecimento teórico desse instrumento é fundamental para sua implementação, pois a falta de entendimento e reconhecimento do conceito e etapas são fatores limitantes para sua aceitação diante da equipe (SILVA AM, et al., 2021).

Nota-se que o desafio para a implementação do PE pode surgir por uma falta de preparação desde a graduação, quando ocorre uma contradição no ensino-aprendizagem. Os acadêmicos às vezes realizam atividades práticas em ambientes de saúde que não aplicam o $P E$ na assistência, fato que inviabiliza a plena compreensão de tal ferramenta (SOUZA JUNIOR DI, et al., 2022).

Estudo realizado em uma instituição de cardiologia apontou que os profissionais que participavam com maior frequência de treinamentos em serviços apresentavam melhor acurácia dos DE. Desta forma, para melhor precisão na identificação dos DE é necessário que os enfermeiros tenham conhecimentos teóricos e práticos dos eventos de enfermagem e familiaridade com a taxonomia de enfermagem (FREITAS NC e CONCEIÇÃO AP, 2018).

O treinamento em serviço favorece uma melhor compreensão em avaliações clínicas e possibilita a aquisição prática de novos conhecimentos, impactando positivamente na qualidade da assistência prestada (FREITAS NC e CONCEIÇÃO AP, 2018). Diante disso, é fundamental incentivar e apoiar os enfermeiros da APS a utilizarem o PE através de cursos de capacitação. Assim, tais profissionais entenderão o PE em sua totalidade, sendo capazes de aplicá-lo. 
Ademais, ressalta-se a importância de se investir em ações de educação permanente com o objetivo de contribuir para o entendimento dos enfermeiros sobre o PE, de maneira a aprimorar as atividades e registros do cuidado. A educação permanente possibilita a aprendizagem efetiva modificando processos de trabalho, apoiando e qualificando as anotações de enfermagem nos prontuários, com a finalidade de assegurar a segurança da equipe de enfermagem e do usuário (MACHADO SKK, et al., 2022).

A sobrecarga de trabalho apresentou-se como segundo fator determinante de interferência para aplicação do PE. Essa questão pode estar correlacionada a diferentes aspectos, dentre eles cita-se a alta demanda de pacientes, sendo o número de pessoas adscritas em uma unidade maior do que o recomendado. Além disso, quando esses usuários buscam atendimento na APS, são direcionados apenas ao enfermeiro, visto que poderiam ser acolhidos por outros membros da equipe, contribuindo ainda mais para essa sobrecarga (BRAGHETTO GT, et al., 2019).

Percebe-se que o excesso de usuários adscritos na unidade gera uma alta demanda espontânea na APS prejudicando diretamente $\mathrm{o}$ acolhimento dos pacientes. $\mathrm{O}$ acolhimento precisa ser feito por meio de avaliações de vulnerabilidade e gravidade para assegurar um atendimento prioritário, ofertando uma escuta qualificada com o objetivo de reconhecer a exata necessidade de saúde de cada indivíduo. Entretanto, o que ocorre diariamente são consultas rápidas similares a urgências devido ao grande número de clientes. Desta forma, os enfermeiros ficam impossibilitados de oferecer um acolhimento desejável e de aplicarem o PE, indo em contrapartida das atribuições do processo de trabalho do profissional da APS, que tem como foco o acolhimento, o vínculo e a longitudinalidade (BRAGHETTO GT, et al., 2019).

Uma questão a ser enfatizada, é em relação ao comportamento flexível dos enfermeiros perante aos demais integrantes atuantes na unidade, o mesmo em determinadas situações assume atividades que seriam de domínio de outros profissionais em detrimento de sua prática privativa. Esse tipo de conduta propicia a sobrecarga e auxilia o aparecimento de pensamentos errôneos acerca do processo de trabalho. Visto isso, o enfermeiro deve se atentar em executar funções de acordo com a Lei do Exercício Profissional, para as quais possui competência técnica e legal (SILVA KM e SANTOS SMA, 2016).

Para mudar esse cenário os integrantes da equipe precisam compreender o seu papel na unidade de saúde. Além disso, deve ser feito a organização de todo o processo de trabalho por meio de definições das competências de cada profissional, com o acolhimento sendo dever de todos os membros, e não somente do profissional de enfermagem, pois é a partir disso que os vínculos entre os usuários e equipe serão fortalecidos. É de suma importância que seja feito essa distribuição para que não gere uma sobrecarga e um desgaste do enfermeiro da APS (BRAGHETTO GT, et al., 2019).

Ainda nesse contexto, percebe-se um quantitativo insuficiente de profissionais atuantes no nível primário. Com isso, começa a emergir uma discrepância entre a demanda dos usuários e a oferta de atendimento, como consequência a assistência de enfermagem fica prejudicada, impossibilitando cada vez mais a realização de atividades que são privativas da categoria (MENDES M, et al., 2020).

Uma alternativa a ser adotada pelos gestores para reduzir esse problema, é o monitoramento dos índices de ausência de profissionais na APS, com isso, poderão ser traçadas medidas para obtenção de um quantitativo adequado de pessoal, a fim de minimizar essa carência. Assim, evita-se a sobrecarga da equipe de enfermagem, bem como garante a prestação de uma assistência segura e de qualidade que seja capaz de suprir as necessidades dos clientes (SILVA KM e SANTOS SMA, 2016).

Outros aspectos relacionados à sobrecarga de trabalho são os excessos de tarefas administrativas e gerenciais desempenhadas pelo enfermeiro, os mesmos são responsáveis tanto pelo preenchimento dos encargos burocráticos da unidade quanto pela supervisão da equipe de enfermagem e dos agentes comunitários de saúde (ACS). Com isso, evidencia-se que o excesso de funções desempenhadas por esses profissionais interfere diretamente na aplicação das etapas do PE, consequentemente a SAE se torna falha (BRAGHETTO GT, et al., 2019). 
No que se refere ao tópico espaço físico, estudos apontam a ausência de locais suficientes para prestação do cuidado. Em determinadas unidades de saúde os consultórios de enfermagem são compartilhados com os demais integrantes da equipe. Em decorrência disso, os atendimentos tendem a ser feitos de maneira mais rápida, priorizando durante a consulta de enfermagem apenas as etapas do PE consideradas relevantes, destacando cada vez mais as falhas na aplicação deste instrumento (SILVA KM e SANTOS SMA, 2016).

Segundo Silva KM e Santos SMA (2016) apesar da Política Nacional de Atenção Básica orientar como infraestrutura necessária a presença de consultório de enfermagem na instituição para a realização das atividades, na prática diária observa-se um cenário diferente. Os locais para atendimento em certos momentos são feitos de maneira improvisada, sem os equipamentos adequados ou, às vezes, os espaços são divididos com outros enfermeiros atuantes na unidade, reforçando ainda mais os fatores que interferem na efetivação do PE (SILVA KM e SANTOS SMA, 2016).

O enfermeiro é responsável por diferentes funções na unidade de saúde, de modo que para realizá-las são necessárias boas condições e locais ideais, para que possa ser prestada uma assistência ampla e de qualidade. Portanto, percebe-se que espaços físicos adequados, além de melhorar os atendimentos, auxilia para a redução da sobrecarga de trabalho dos profissionais, promovendo satisfação tanto para equipe quanto para os usuários da APS (MENDES M, et al., 2020).

Quanto a desvalorização do profissional enfermeiro, nota-se que no Brasil a enfermagem vem sofrendo manutenção de jornada extensiva de trabalho, perda de direitos, baixo salário e vínculos empregatícios frágeis. Isso revela a deterioração das condições trabalhistas e a perda de direitos para tal categoria (SOUZA TO, et al., 2021).

Uma pesquisa realizada com acadêmicos de enfermagem evidenciou três tipos de desvalorização da enfermagem: a midiática, a política e a financeira. Percebe-se a predominância do modelo biomédico nos canais de comunicação, pois tal categoria ainda é vista como submissa a equipe médica. Ademais, existe grande diferença salarial quando analisada pelo trabalho desenvolvido, gerando desvalorização da profissão (FERREIRA JCSC, et al., 2020).

No contexto da atual pandemia de COVID-19, ocorreram protestos da categoria por valorização profissional, instituições organizativas da profissão, como o COFEN, realizaram campanhas em prol do reconhecimento profissional dos enfermeiros. Destaca-se o "Ato Virtual pela Enfermagem aos profissionais mortos na pandemia", realizado em 12 de maio de 2020, sendo mobilizado, via internet, publicação de mensagens, vídeos e imagens com o objetivo de dar visibilidade à Enfermagem (SOUZA TO, et al., 2021).

A enfermagem almeja visibilidade política e social. Entretanto, atualmente nota-se que ainda há desconhecimento e desinformação a respeito da verdadeira atuação do profissional de enfermagem na equipe multidisciplinar de saúde. Sendo assim, para existir valorização e reconhecimento é necessário registrar, publicar e divulgar habilidade técnica, científica e articulação política com ações proativas, especialmente, nas situações caóticas nas quais a enfermagem assume a linha de frente (LUZ GOA, et al., 2020).

Dentre os pontos que tiveram menor prevalência, mas mesmo assim se apresentou como fator de interferência para a aplicação do PE, podemos citar a dificuldade do enfermeiro em encontrar um DE adequado para o problema de saúde identificado. Diante do tópico exposto, nota- se que a segunda etapa do PE não está sendo utilizada corretamente, pois em alguns momentos o profissional demostra dúvidas em definir o diagnóstico de acordo com o quadro clínico do paciente, como consequência ocorre um prejuízo na efetivação dessa etapa e das fases subsequentes do processo (COSTA AS, et al., 2018).

Um estudo produzido sobre o uso da taxonomia pelos enfermeiros durante a prestação da assistência, evidenciou que grande parte dos profissionais relataram não fazer o uso de nenhuma classificação de diagnóstico. Os que utilizam a taxonomia, citaram a Classificação Internacional para Prática de Enfermagem (CIPE) e North American Nursing Diagnosis Association (NANDA-I), sendo essa última ferramenta a mais conhecida e estudada durante o período de graduação. Houve aqueles que confundiram com os sistemas de patologias e programas de atendimento (COSTA AS, et al., 2018). 
A etapa de DE está diretamente relacionada ao uso de Sistemas de Linguagem Padronizada (SLP), pois o uso de termos padronizados permite a realização de uma lógica precisa, evitando inconsistências no julgamento clínico. Os SLP são instrumentos que colaboram com o raciocínio diagnóstico e favorecem a comunicação de qualidade, o que contribui para que a prática de enfermagem seja visível, efetiva e quantificável (MACHADO SKK, et al., 2022). Diante disso, é possível perceber ausência de conhecimento em relação a segunda etapa do processo, e o impacto que isso pode ocasionar na continuidade do cuidado de enfermagem (COSTA AS, et al., 2018).

\section{CONSIDERAÇÕES FINAIS}

Os resultados do presente artigo evidenciaram diversos aspectos que dificultam a aplicação do PE pelos enfermeiros na APS. Diante dessas informações, é possível traçar planos e estratégias para solucionar tais adversidades, como por exemplo, aproximação da teoria com a prática durante a graduação, a fim de que os discentes tenham maior familiaridade com o PE, compreendam sua relevância e o insira na sua rotina profissional. Dessa forma, ocorrerá maior estímulo e incentivo para o uso de tal instrumento que é tão importante para o desenvolvimento científico da profissão.

\section{REFERÊNCIAS}

1. ADAMY EK, et al. Formação de enfermeiros sobre anamnese e exame físico. Journal of Nursing and Health, 2016;6(2): 334-345.

2. AMARAL IBST, SILVA ALA. Consulta do enfermeiro na estratégia saúde da família: Um recorte do Rio de Janeiro. Revista Online de Pesquisa: Cuidado é Fundamental, 2021;13: 227-233.

3. BEZERRIL MS, et al. Ensino do processo de enfermagem segundo os vídeos do youtube: estudo descritivoexploratório. Online Brazilian Journal Of Nursing, 2021; 20: 1-12.

4. BRAGHETTO GT, et al. Dificuldades e facilidades do enfermeiro da Saúde da Família no processo de trabalho. Cadernos Saúde Coletiva, 2019; 27(4): 420-426.

5. BRASIL. Portaria $n^{\circ}$ 2.436, de 21 de setembro de 2017. 2017. Disponível em: https://bvsms.saude.gov.br/bvs/saudelegis/gm/2017/prt2436_22_09_2017.html. Acessado em: 25 de maio de 2020.

6. CONSELHO FEDERAL DE ENFERMAGEM (COFEN). Resolução COFEN n. 358, de 15 de outubro de 2009.2009. Disponível em: http://www.cofen.gov.br/resoluo-cofen-3582009_4384.html. Acessado em: 07 de janeiro de 2021.

7. CORREA VAF, et al. Cuidado do enfermeiro na Estratégia Saúde da Família: práticas e fundamentações teóricas. Revista Brasileira de Enfermagem, 2018; 71: 2932-2939.

8. COSTA AS, et al. O processo de Enfermagem na Atenção Básica em um munícipio de Alagoas, Brasil. Revista de Enfermagem e Atenção à Saúde, 2018; 7(1): 143-151.

9. FERREIRA JCSC, et al. Onde está a enfermagem? A (in)visibilidade desta categoria profissional nos meios de comunicação. Enfermagem em Foco, 2020; 11(2): 50-56.

10. FREITAS NC, CONCEIÇÃO AP. Acurácia dos diagnósticos de enfermagem de uma instituição de cardiologia. Revista de Enfermagem Ufpe On Line, 2018; 12(10): 2727-2736.

11. GANDOLFI M, et al. Sistematização da assistência de enfermagem: da teoria ao cuidado integral. Revista de Enfermagem UFPE Online, 2016; 10(4): 3694-3703.

12. GOMES PAR, et al. Prontuário eletrônico do cidadão: instrumento para o cuidado de enfermagem. Revista Cuidado é Fundamental Online, 2019; 11(5): 1226-1235.

13. KAHL C, et al. Ações e interações na prática clínica do enfermeiro na atenção primária à saúde. Revista da Escola de Enfermagem da USP, 2018; 52.

14. LOWEN IMV, et al. Inovação na prática assistencial do enfermeiro: ampliação do acesso na atenção primária. Revista Brasileira de Enfermagem, 2017; 70(5): 945-951.

15. LUZ GOA, et al. Registro de enfermagem perante a COVID-19: contribuições para assistência, ensino, pesquisa e valorização da enfermagem. Estima, Brazilian Journal Of Enterostomal Therapy,2020; 1-3.

16. MACHADO SKK, et al. Aplicabilidade do processo de enfermagem na atenção hospitalar: interface com as melhores práticas. Revista de Enfermagem da UFSM, 2022; 12: 1-18.

17. MARCHIORI GRS, et al. Saberes sobre o processo de enfermagem no banco de leite humano. Texto ContextoEnfermagem,2018; 27(2): 1-10.

18. MATA LRF, et al. Consultas mais frequentes respondidas pelo conselho regional de enfermagem de Minas Gerais: atuação da câmara técnica. Revista Oficial do Conselho Federal de Enfermagem, 2019;10(1): 18-23. 
19. MENDES M, et al. Cargas de trabalho na Estratégia Saúde da Família: interfaces com o desgaste dos profissionais de enfermagem. Revista da Escola de Enfermagem da USP, 2020; 54: 1-9.

20. MOTA BAM, et al. Efetividade da consulta de enfermagem na adesão ao tratamento da hipertensão arterial sistêmica. Revista Salud pública, 2019; 21(3).

21. PIOVESAN G, et al. Qualidade da atenção primária na perspectiva de profissionais: saúde de crianças e adolescentes com HIV. Texto Contexto-Enfermagem, 2017; 26(2): 1-11.

22. RIBEIRO GC, PADOVEZE MC. Sistematização da Assistência de Enfermagem em unidade básica de saúde: percepção da equipe de enfermagem. Revista da Escola de Enfermagem da USP, 2018; 52: 1-7.

23. ROSA APL, et al. Gestão do cuidado à mulher na atenção primária: estratégias para efetivação do processo de enfermagem. Revista Oficial do Conselho Federal de Enfermagem,2020; 11(1): 93-98.

24. SANTOS KC, et al. Atenção à saúde do homem: construção e validação de instrumento para a consulta de enfermagem. Revista Brasileira de Enfermagem, 2020; 73(3): 1-10.

25. SIEGA CK, et al. Vivências e significados da consulta do enfermeiro em puericultura: análise à luz de Wanda Horta. Revista de Enfermagem da UFSM, 2020; 10(65): 1-21.

26. SILVA KM, SANTOS SMA. Consulta de enfermagem na estratégia saúde da família: realidade de um distrito sanitário. Revista de Enfermagem da UFSM, 2016; 6(2): 248-258.

27. SILVA AM, et al. Percepções dos enfermeiros acerca da implementação do processo de enfermagem em unidade intensiva. Revista Gaúcha de Enfermagem, 2021; 42: 1-7.

28. SILVEIRA V, et al. Sistematização da assistência de enfermagem na saúde da família: percepção dos acadêmicos de enfermagem. Revista de Enfermagem UFPE Online, 2016; 10(11): 3892-3900.

29. SOMARIVA VCA, et al. Percepções das equipes de enfermagem na atenção básica frente à sistematização da assistência de enfermagem. Revista Oficial do Conselho Federal de Enfermagem, 2019;10(4):142-147.

30. SOUSA LMM, et al. Revisões da Literatura Científica: tipos, métodos e aplicações em enfermagem. RPER, 2018; 1(1): 45-54.

31. SOUZA TO, et al. Enfermagem e visibilidade na pandemia da COVID-19: monitoramento de mídia social. Revista Baiana de Enfermagem, 2021; 35: 1-13.

32. SOUZA JUNIOR DI, et al. O enfermeiro vivenciando a intencionalidade como significado na implementação do processo de enfermagem. Revista de Pesquisa Cuidado é Fundamental, 2022.

33. ZANATTA EA, et al. Consulta de enfermagem em puericultura à criança haitiana: dificuldades e possibilidades. Revista Baiana de Enfermagem, 2020; 34. 